\title{
Parameters Online Detection and Model Predictive Control during the Grain Drying Process
}

\author{
Lihui Zhang, ${ }^{1,2}$ Helei Cui, ${ }^{1}$ Hongli Li, ${ }^{1}$ Feng Han, ${ }^{1}$ Yaqiu Zhang, ${ }^{1}$ and Wenfu Wu ${ }^{1}$ \\ ${ }^{1}$ School of Biological and Agricultural Engineering, Jilin University, Changchun 130022, China \\ ${ }^{2}$ School of Electrical and Electronic Information Engineering, Jilin Jianzhu University, Changchun 130118, China \\ Correspondence should be addressed to Wenfu Wu; dzzlhh@sina.com
}

Received 4 March 2013; Revised 12 May 2013; Accepted 12 May 2013

Academic Editor: Shane Xie

Copyright (c) 2013 Lihui Zhang et al. This is an open access article distributed under the Creative Commons Attribution License, which permits unrestricted use, distribution, and reproduction in any medium, provided the original work is properly cited.

\begin{abstract}
In order to improve the grain drying quality and automation level, combined with the structural characteristics of the crossflow circulation grain dryer designed and developed by us, the temperature, moisture, and other parameters measuring sensors were placed on the dryer, to achieve online automatic detection of process parameters during the grain drying process. A drying model predictive control system was set up. A grain dry predictive control model at constant velocity and variable temperature was established, in which the entire process was dried at constant velocity (i.e., precipitation rate per hour is a constant) and variable temperature. Combining PC with PLC, and based on LabVIEW, a system control platform was designed.
\end{abstract}

\section{Introduction}

Drying is an important part of the grain processing after harvest, while drying monitoring and control are directly related to the management of the drying operation, the drying efficiency, and the quality of the dried grain. At present, in the actual production, the degree of automation and informatization of drying monitoring is not high; it is difficult to accurately monitor the whole drying process, which affects the accuracy of the automatic control. Grain drying process is a typical multivariable, time-varying, nonlinear, and large delay industrial production process; the drying process is difficult to achieve accurate automatic control [1].

In this paper, specific to the drying process of the cross-flow circulation grain dryer, a drying model predictive control system was set up. Using the virtual instrument technology, an automatic detection and control system for the circulation grain dryer were developed, to detect and control the influencing factors in the drying process, in order to achieve the automation of the control process.

\section{Process Design of the Dryer}

Shown in Figure 1 is the flow diagram of our developed crossflow circulation grain dryer.
Generally, the cross-flow circulation grain dryer adopts drying and retarding processes. In the cross-flow phase, the hot air flows vertically through the top-down grain layer, removes the excess moisture; while in the retarding phase, mainly the grain moisture gradient decreases, and the particles internal moisture migrates outward, thus ensuring the grain quality [2].

When operation, the grain in the hopper is transferred to the top of the dryer by an grain elevator, then will be evenly thrown into the up storage by the up auger, next moves down to the drying section by gravity. The speed is controlled by the revolution of the discharge motor. In the drying section, the hot air passes the grain layers through hot air piping and air plate. After drying, the moist air is discharged from the rear through duct by a centrifugal blower, and thus the grain is dried. Then the dried grain enters into the down storage for retarding, and the retarded grain enters into the down drying section to continue drying, and thus a dryingretarding-drying-retarding process forms. The dried grain is discharged to the down storage by the discharge auger. At the down storage, by a chain driven blade, the grain will be transferred into the feed hopper and will be conveyed again to the top of the dryer by the elevator, again and again, until the grains reach the target moisture content after discharge. 


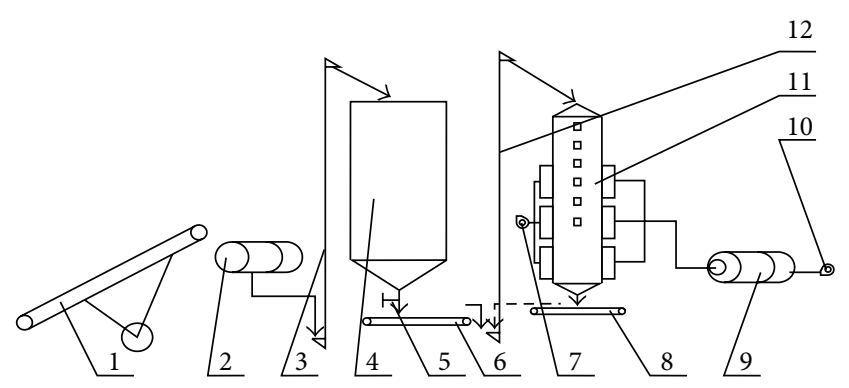

FIgURE 1: Dryer process flow diagram. (1) belt conveyor, (2) precleaner, (3) bucket elevator, (4) warehouse for unprocessed grain, (5) manual or pneumatic gate, (6) flat belt conveyor, (7) cooling fan, (8) chain conveyor, (9) heat source device, (10) hot air blower, (11) drying tower, (12) bucket elevator.

\section{Parameters Online Detection}

The system adopts distributed control, as shown in Figure 2. The system consists of a host (Industrial PC), PLC, ambient temperature and moisture sensors, temperature sensors, resistive online moisture meter, actuators, and the relevant instruments. The main parameters include hot air temperature, grain temperature, offgas temperature, ambient temperature and humidity, the raw grain moisture and dryer exit grain moisture; the operative part includes PLC, inverter, and other peripheral lines.

Data exchange between host and temperature instruments, moisture meter and actuators through the RS232/RS485 converter module I-7520 serial communication data bus. On the one hand, the host receives data of lower machine from the temperature instruments and moisture sensors; on the other hand, the host sends control words to lower machine such as PLC, temperature instruments, and moisture sensors, to make corresponding controls $[3,4]$.

Figure 3 shows sensors and controllers layout, including duct hot air temperatures $(T 1, T 2)$, hot air temperature sensors $(T 3, T 4)$, grain temperature sensors $(T 5, T 6)$, grain moisture meters (M1, M2), grain level sensors (L1, L2), actuators (including PLC (C1), hot air speed inverter (C2), and grain discharge speed inverter (C3)), and ambient temperature and moisture sensors $(T 7, \mathrm{H} 1)$. PLC, inverter, and ambient temperature and moisture sensors are not shown in the layout diagram.

Moisture online detection is the key in the grain drying control system. Our self-developed online moisture meters, adopted by the system, were installed in the inlet and outlet elevators, respectively.

The resistive online moisture meter consists of a host and a moisture sensor. The moisture sensor is shown in Figure 4. For the moisture meter, according to the measured resistance in the relationship with the moisture content when wheels crush the grain, by measuring the resistance to online measure the grain moisture level. The output resistance is also associated with parameters such as grain temperature, varieties, and maturity. With automatic and manual control modes, the moisture meter has functions of grains switching, moisture setting, moisture correction, and temperature compensation. The target moisture value can be preset. The meter will respond upon arrival at this value to avoid over and insufficient drying. The meter can be intermittent cycle automatic start, timing start online monitoring moisture, with high sensitivity and good stability.

\section{Cross-Flow Dryer Model Predictive Control}

The characteristics of complexity, time-varying, hysteresis, and nonlinearity in grain drying bring a lot of difficulties to the traditional control applications. MPC (model predictive control) is a composite optimal control algorithm based on the model, by rolling implementation combined with feedforward forecast and feedback correction. Its control output can track set-points, particularly effective for nonlinear and large delay control [5]. Some foreign researchers developed a new type of model predictive controller for cross-flow dryer, which was successfully applied to actual production [6].

The MPC system structure of the cross-flow circulation grain dryer is shown in Figure $5[6,7]$, including a feedforward loop (consists of process model, reverse process model, and grain discharge speed optimizer) and a feedback loop (parameter estimation/correction). The system has the following input parameters: grain initial moisture, grain target drying moisture, and dry air temperature. The parameter that needs to be optimized is the grain discharge speed.

4.1. Drying Process Model. Assume that the medium temperature can be precisely controlled and the ambient temperature and humidity are relatively stable. The grain is divided into a number of thin layer to analyze, in order to set up grain drying control model. According to the structure of the drying section of the cross-flow circulation grain dryer and hot air drying characteristics, the grain columns of the dryer section can be seen as a collection of a series of rectangles differential unit in the vertical direction [7], as shown in Figure 6. For each rectangle differential unit, a rectangular width is the thickness of the drying section; that is, the thickness of the grain layer, and the height is the differential $\Delta Y$ or $d y$ of length of the drying section; it may be sufficiently small. Also assume that the hot air temperature and humidity change or the difference in the $Y$ direction is negligible.

Constant velocity (equal precipitation rate) variable temperature control and target control are combined, to control the grain precipitation rate within less than $1.5 \% / \mathrm{h}$. Compare target moisture with the measured moisture and stop drying after the safe moisture or the final set value arrived.

First we assumed the following parameters: initial moisture $M_{0}$, target moisture $M_{T}$, single cycle target precipitation rate $\eta \%$, and grain discharge speed $G ; \delta$ is a moisture difference in order to achieve a safe moisture. The model is derived as follows.

The grain target drying moisture:

$$
\left|M_{0}-M_{T}\right| \leq \delta \text {. }
$$

Cycles to achieve the target moisture:

$$
n=\frac{M_{T}-M_{0}}{\eta} \text {. }
$$




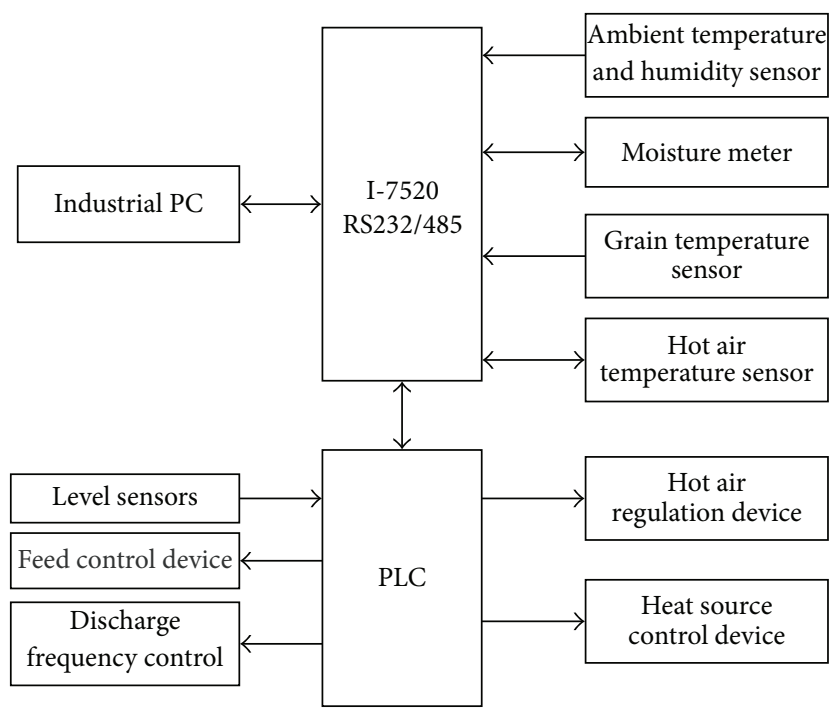

FIGURE 2: The composition block diagram of the control system.

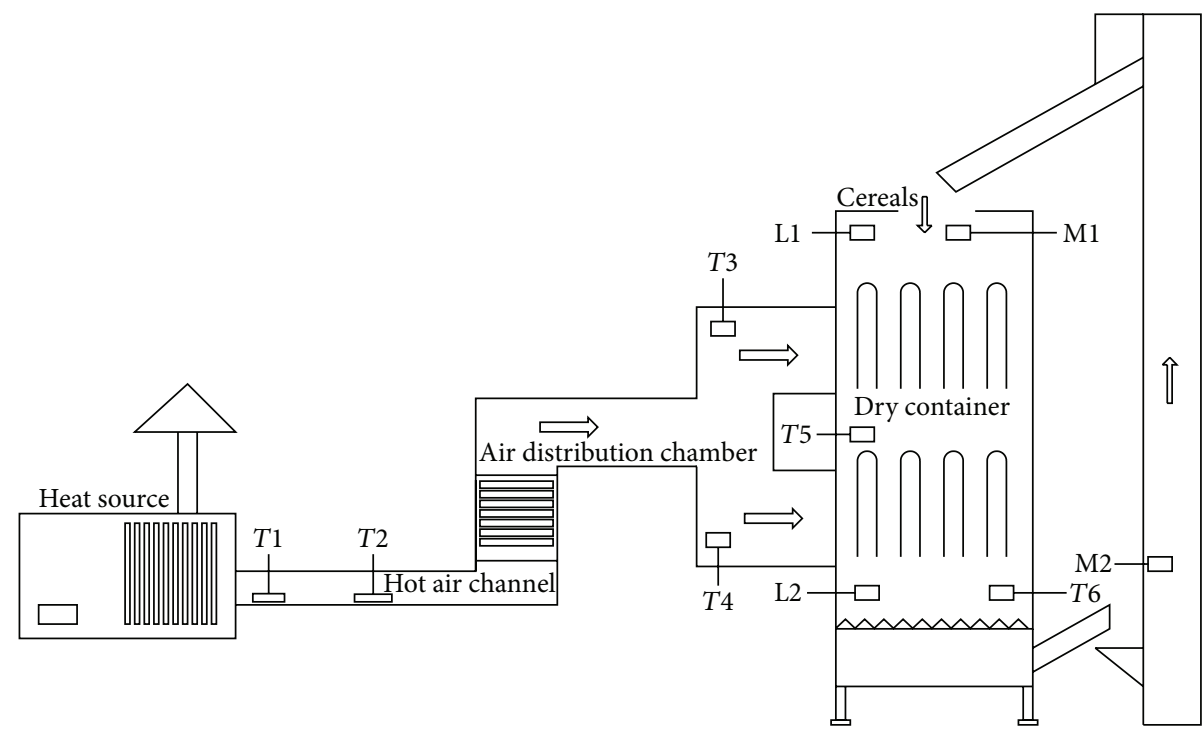

FIGURE 3: Sensors and controllers layout.

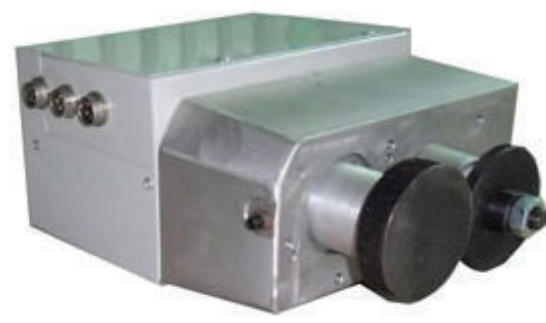

Figure 4: Moisture sensor.

During the entire grain drying process, the moisture changes of the grain over time is as follows: $M_{0}, M_{1}, M_{2}, \ldots, M_{i}, \ldots, M_{n}$. Then there is the following relationship:

$$
\begin{aligned}
M_{1}-M_{0} & =M_{2}-M_{1} \\
& =\cdots=M_{i+1}-M_{i}=\cdots=M_{n+1}-M_{n} .
\end{aligned}
$$

Grain drying model PAGE:

$$
\begin{gathered}
\mathrm{MR}_{i+1}=\frac{M_{i+1}-M_{e, i}}{M_{i}-M_{e, i}}=\frac{M_{i}-\eta-M_{e, i}}{M_{i}-M_{e, i}}, \\
\mathrm{MR}_{i+1}=\exp \left[-k_{i} \Delta_{i+1}^{n_{i}}\right], \\
k_{i+1}=k_{i}=0.01313 \cdot \exp \left(0.0175 T_{i}\right), \\
n_{i+1}=0.748+0.163 \cdot V_{i+1} .
\end{gathered}
$$




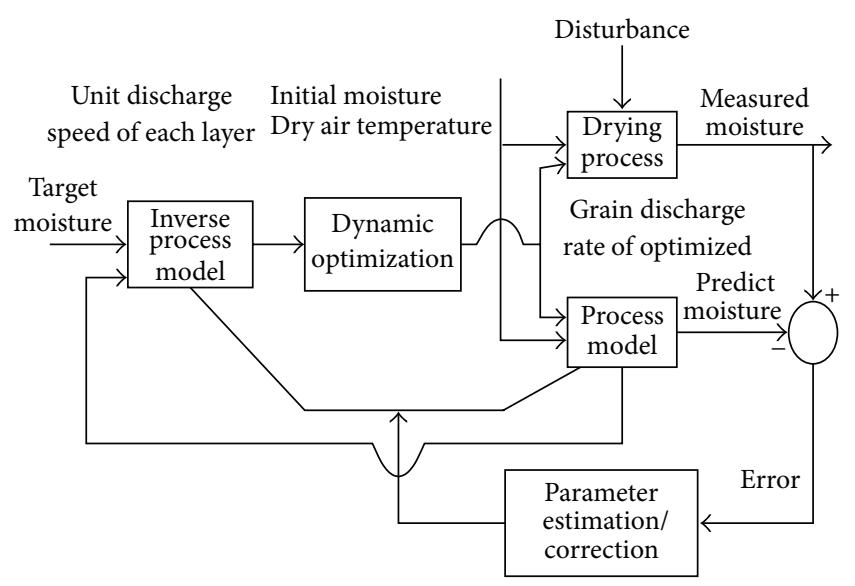

Figure 5: The MPC system structure of the cross-flow circulation grain dryer.

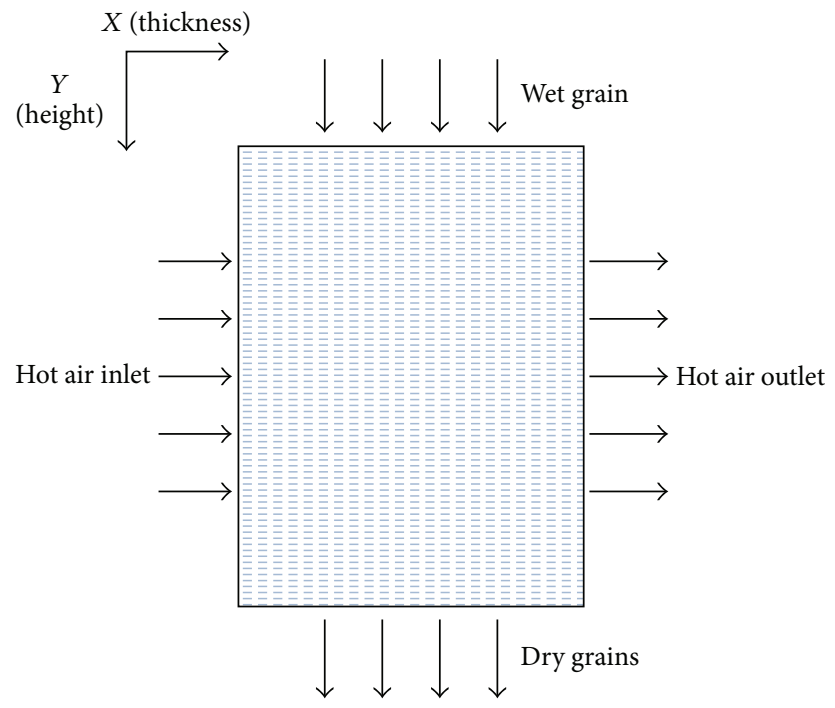

Figure 6: Schematic of cross-flow drying.

During the drying process, it is assumed that the inlet grain moisture of a unit layer is $M_{j}$, the outlet grain moisture is $M_{j+1}, \Delta Y$ is the height of the unit layer, and the dryer discharge speed at the time is $G_{g}$; then

$$
\begin{gathered}
\Delta_{t}=\frac{\Delta Y}{G_{g}}, \\
M_{j+1}=\left(M_{j}-M_{e, i}\right) \mathrm{MR}_{i+1}+M_{e, i}, \\
\mathrm{MR}_{i+1}=\exp \left[-k_{i}\left(\frac{\Delta Y}{G_{g}}\right)^{n_{i}}\right], \\
M_{e, i}=\sqrt{\frac{\ln \left(1-\mathrm{RH}_{i}\right)}{3.82 \times 10^{-5}\left(1.8 T_{i}+82\right)}} .
\end{gathered}
$$

In which, $T_{i}$ is drying hot air temperature $\left({ }^{\circ} \mathrm{C}\right), M_{i}$ is the grain moisture after drying a certain period time (dry basis), $\mathrm{MR}$ is the grain moisture ratio, $M_{e}$ is the equilibrium moisture content of the grain (dry basis), $k$ is a constant, $n$ is the number of the grain thin layer, $\mathrm{RH}$ is the hot air relative humidity, $V_{i}$ is the speed of hot $\operatorname{air}(\mathrm{m} / \mathrm{s})$, and $i$ is the sample number, $i=1,2, \ldots, n$.

Thus using (6), (7), and (8), all unit layers of the entire drying section can be solved, and the final discharge moisture from the cross-flow dryer and the grain moisture distribution within the whole drying section were further obtained.

4.2. Reverse Drying Process Model. For drying production, generally the grain initial and target moisture are known to set the appropriate parameters in order to achieve the purpose of drying.

When the grain discharge moisture $M_{f}$ is set, the reverse drying process model can be used to calculate the dryer theoretical grain discharge speed $G_{0}$. Grain moisture at $Y$ away from the discharge outlet is $M_{y}$. The grain discharge speed $G_{y}$ will be obtained if $M_{y}$ reaches the target moisture as follows:

$$
\begin{gathered}
G_{y}=\frac{Y}{e \sqrt[N]{\ln \left[-(1 / k) \ln \left(\left(M_{f}-M_{e}\right) /\left(M_{y}-M_{e}\right)\right)\right]}}, \\
M_{e}=\sqrt{\frac{\ln (1-\mathrm{RH})}{3.82 \times 10^{-5}(1.8 T+82)}} .
\end{gathered}
$$

4.3. The Grain Discharge Speed Optimization. Due to the presence of environment interference, as well as the model mismatch as a result of the simplified model, the theoretical grain discharge speed of each unit layer calculated by (9) may be unequal. But in the actual drying process, all unit layers in the drying section can only move down at the same discharge rate. Therefore, in the MPC control system, a dynamic optimizer is needed to optimize the calculation of the grain discharge speed, in order to obtain an optimal value. In this system, zero average error method was applied [8].

Assume that the grain moisture at the $j$ th unit layer is $M_{j}$; in the next $j$ sampling periods, the real grain discharge speed before the $j$ th unit layer reaches the outlet is $G_{g}$; then the moisture of these grains when they arrive at the outlet can be estimated as follows:

$$
M_{f j}=M_{j}-\alpha \frac{j \Delta Y}{G_{g}},
$$

where $M_{f j}$ is the grain final moisture; the value of lay unit $j$ is $1,2,3, \ldots, n$, starting from inlet to the outlet; $j \Delta Y / G_{g}$ is the time required of the grain from the current unit layer to the discharge outlet; $\alpha$ is a constant, dependent on the drying model and conditions.

Assuming that the target moisture after drying is $M_{t}$ and the ideal dryer discharge speed is $G_{g j}$, then

$$
M_{t}=M_{j}-\alpha \frac{j \Delta Y}{G_{g j}}
$$

In the next sampling period, when the actual grain discharge speed $G_{g}$ and the ideal grain discharge speed $G_{g j}$ 


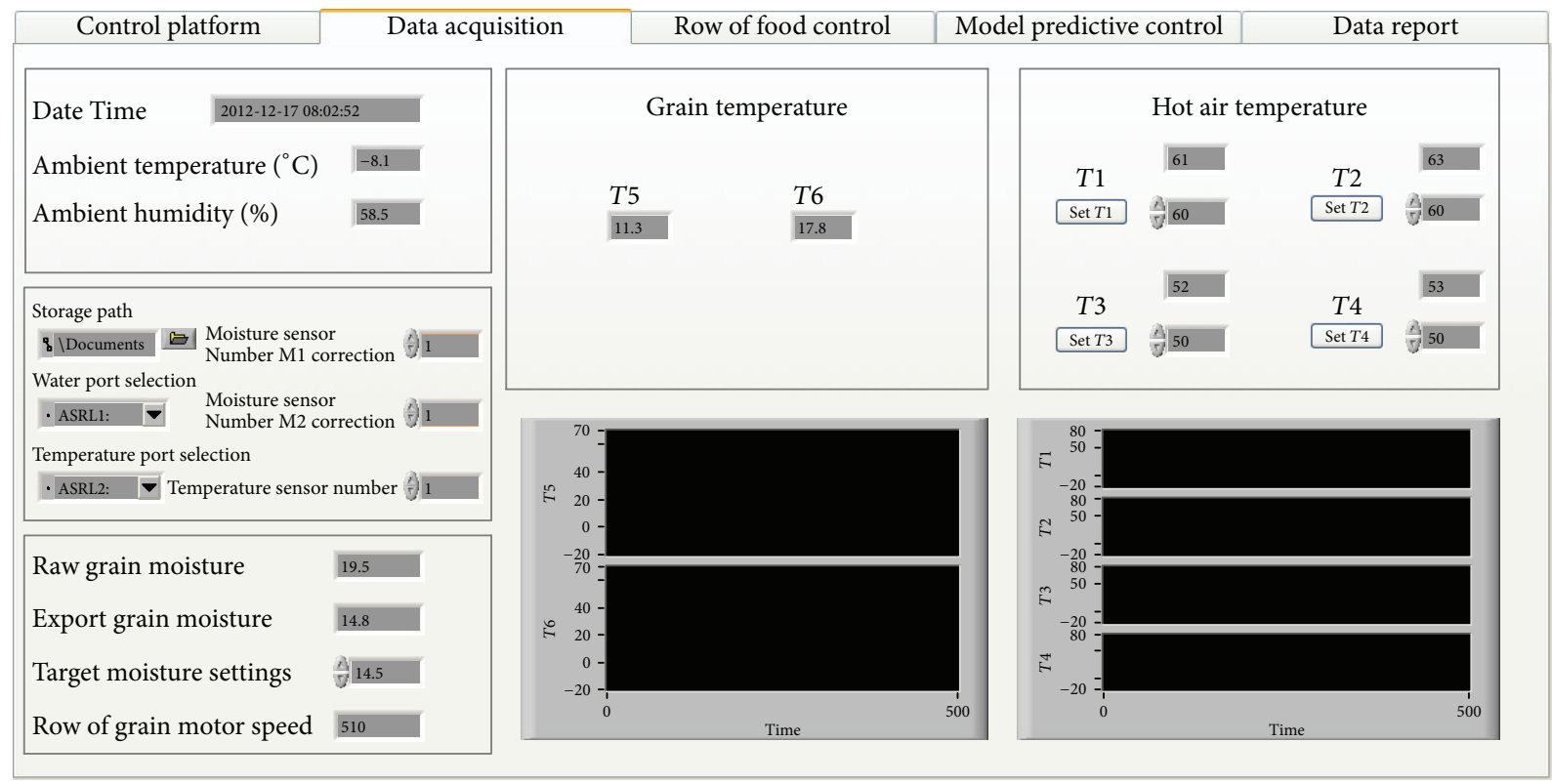

FIGURE 7: Front panel of data display.

are not equal, then there will be deviation between the final grain moisture and the target moisture; the result is

$$
e_{j}=M_{f j}-M_{t}=\alpha \Delta Y\left(\frac{j}{G_{g j}}-\frac{j}{G_{g}}\right) .
$$

This error is only for one unit layer, so for the whole grain column, the average error is

$$
e=\frac{1}{n} \sum_{j=1}^{n} e_{j}=\frac{\alpha \Delta Y}{n} \sum_{j=1}^{n}\left(\frac{j}{G_{g j}}-\frac{j}{G_{g}}\right) .
$$

Assumed $e=0$, the real optimized grain discharge speed can be

$$
G_{g}=\frac{n(n+1)}{2 \sum_{j=1}^{n}\left(j / G_{g j}\right)} .
$$

4.4. Feedback Correction of the Control Model. The process model error is defined as the difference between the measured outlet grain moisture and the target grain moisture. If the error is not zero, drying constant $k$ of the process and reverse process models is corrected using the empirical coefficient $\gamma$. In the feedback loop, parameter estimation/correction is as follows [9]:

$$
\begin{gathered}
k^{m}=\gamma k, \\
\gamma=\gamma_{l}+\beta \ln \frac{M_{f p}}{M_{f m}},
\end{gathered}
$$

wherein $k^{m}$ is the drying constant after correction. $\gamma$ is an experience correction coefficient, obtained from the formula
(16), which is the current correction coefficient. $\gamma_{l}$ is a correction coefficient of a previous cycle (default is 0.8 ). $\beta$ is a filter coefficient, determined by experiment, by adjusting the controller. $M_{f p}$ is the predicted grain moisture, and $M_{f m}$ is the actual measured moisture.

\section{System Software Design}

Graphical programming language LabVIEW is used for design of the system operation platform. The modular structure design is adopted, and system software consists of modules of drying model predictive control system, detection and control of hot air temperature, grain temperature detection, grain moisture detection and correction, and grain discharge speed control. Specifically, it is divided into the system initialization module, the data acquisition and display module, the data storage and processing module, and the system control module. By the system initialization module, the control system parameters can be set and modified. Data acquisition and display module will realize real-time data collection from instrument transmission and visually display in the man-machine interface. Data storage and processing module will store the collected data, while the collected data is graphed on the man-machine interface. According to data values and graphs for each time, the system control module is in accordance with the model predictive control algorithm for real-time automatic control. The data acquisition interface is shown in Figure 7 [10].

The model predictive control system was test simulated in LabVIEW. The simulation results show that, when the grain feed moisture and the drying air temperature are within a certain range of variation, by the control of the MPC, the grain discharge moisture closes to the set target moisture. 


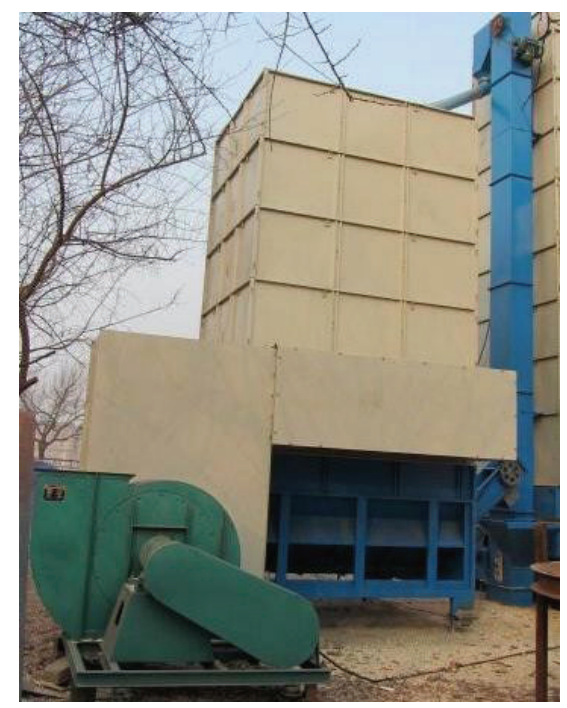

FIGURE 8: Application site.

TABLE 1: Initial Conditions of the Drying Test (average value).

\begin{tabular}{lccc}
\hline Item & $1.5 \% / \mathrm{h}$ & $1.0 \% / \mathrm{h}$ & $0.5 \% / \mathrm{h}$ \\
\hline Ambient temperature $/{ }^{\circ} \mathrm{C}$ & -8.0 & -7.2 & -9.1 \\
Ambient humidity $\%$ & 59 & 65 & 55 \\
Raw grain moisture/\% & 19.3 & 19.5 & 19.4 \\
\hline
\end{tabular}

\section{System Application}

In November 2012, production test experiment of drying rice was carried out on the system. The application indicates that the system has the advantages of simple hardware structure, high precision, high on-site anti-interference ability, high degree of automation, and work friendly interface. Figure 8 shows the application site of the cross-flow circulation grain dryer. We tested working conditions of the circulation dryer at precipitation rates of $1.5 \% / \mathrm{h}, 1.0 \% / \mathrm{h}$, and $0.5 \% / \mathrm{h}$. The initial drying conditions are shown in Table 1. Figure 9 shows the outlet grain moisture curve at the three drying precipitation rates. From the curve we observed that the precipitation rate is approximately constant velocity, and outlet rice moisture detection is more accurate. Figure 10 is the hot air temperature variation curve at the three drying precipitation rates. Under the premise of maintaining the equal precipitation rate during the whole drying process, using the constant velocity variable temperature prediction model to control the temperature of hot air, hot air temperature fluctuations are little and can be better controlled. When the constant speed precipitation rate is $1.5 \% / \mathrm{h}$, the average hot air temperature of $68^{\circ} \mathrm{C}$ is needed; the higher the temperature of the drying medium, the poorer the quality of grain after drying. When the constant speed precipitation rate is $0.5 \% / \mathrm{h}$, the dried rice quality is good, but the drying time and energy consumption are high. Comprehensive comparison shows that constant velocity precipitation rate of $1.0 \% / \mathrm{h}$ has a good dried rice quality, the highest drying efficiency, and the lowest energy consumption.

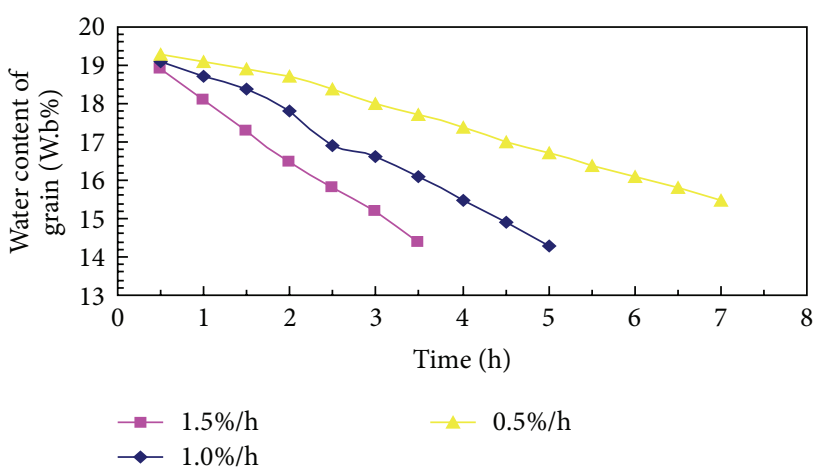

FIGURE 9: Outlet grain moisture at different drying precipitation rates.

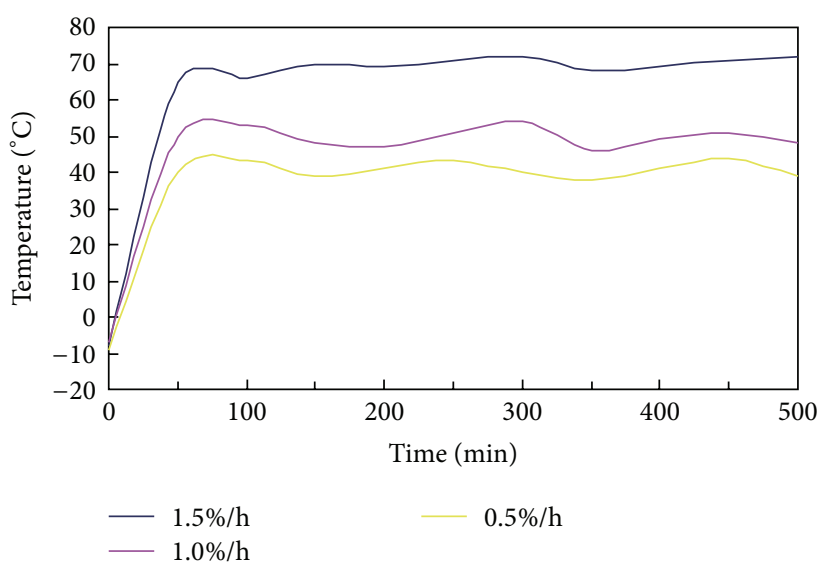

FIGURE 10: Hot air temperature changes at different drying precipitation rates.

\section{Conclusion}

According to the structural characteristics of the cross-flow circulation grain dryer, process, and system control objectives and requirements, a grain drying parameters detection system was established. The system can automatically detect and display the parameters of the grain temperature, hot air temperature, and grain moisture. A grain drying process model predictive control system was established, and the model predictive control combined moisture model prediction with the advantages of the control function of grain discharge speed optimization, can compensate for changes of grain drying conditions, and is particularly effective for nonlinear and large delay drying control. A constant velocity variable temperature of drying process predictive control model was established. System software was developed using LabVIEW, to achieve automatic control of the grain drying process and to better guarantee the quality of the grain drying.

\section{Conflict of Interests}

In this paper, "LabVIEW software" is only used in the design of the system software for academic research, and the authors 
have no interest relationship and conflict with LabVIEW company (National Instruments).

\section{Acknowledgment}

This work is supported by the High-Tech Research and Development Program of China (863 Program) (2006AA10Z256).

\section{References}

[1] X. Liu, Complexity Analysis and Process Control for Grain Drying, Jilin University, Changchun, China, 2003.

[2] Y. Zhang, Moisture Detection and Automatic Control of Grain Drying Process, Jilin University, Changchun, China, 2012.

[3] F. Han, W.-F. Wu, Y.-Q. Zhang, and H. Zhu, "Automatic control system of grain dryer based on virtual instrument," Journal of Jilin University, vol. 39, no. 3, pp. 643-647, 2009.

[4] J. Zhang, Y. Lu, H. Liu, and X. Tang, "On-line measurement and intelligent prediction control of grain parameters in drying process," Transactions of the Chinese Society of Agricultural Machinery, vol. 34, no. 2, pp. 50-53, 2003.

[5] H.-O. Wang, Z.-C. Hu, K. Tu, F.-L. Ji, Y.-Q. Chen, and L.-L. $\mathrm{Hu}$, "Application research of model-predictive control in grain drying," Drying Technology \& Equipment, vol. 6, no. 6, pp. 267271, 2008 .

[6] Q. Liu and F. W. Bakker-Arkema, "A model-predictive controller for grain drying," Journal of Food Engineering, vol. 49, no. 4, pp. 321-326, 2001.

[7] Q. Liu and F. W. Bakker-Arkema, "Automatic control of crossflow grain dryers, part 1: development of a process model," Journal of Agricultural Engineering Research, vol. 80, no. 1, pp. 81-86, 2001.

[8] Q. Liu and F. W. Bakker-Arkema, "Automatic control of crossflow grain dryers, part 2: design of a model-predictive controller," Journal of Agricultural Engineering Research, vol. 80, no. 2, pp. 173-181, 2001.

[9] Q. Liu and F. W. Bakker-Arkema, "Automatic control of crossflow grain dryers, part 3: field testing of a model-predictive controller," Journal of Agricultural Engineering Research, vol. 80, no. 3, pp. 245-250, 2001.

[10] J. Travis and J. Kring, LabVIEW for Everyone Graphical Programming Made Easy and Fun, Publishing House of Electronics Industry, 3rd edition, 2008. 


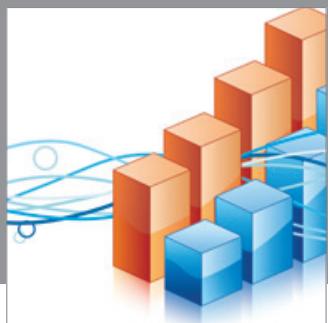

Advances in

Operations Research

mansans

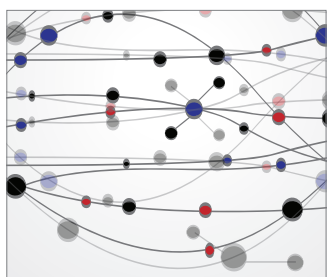

The Scientific World Journal
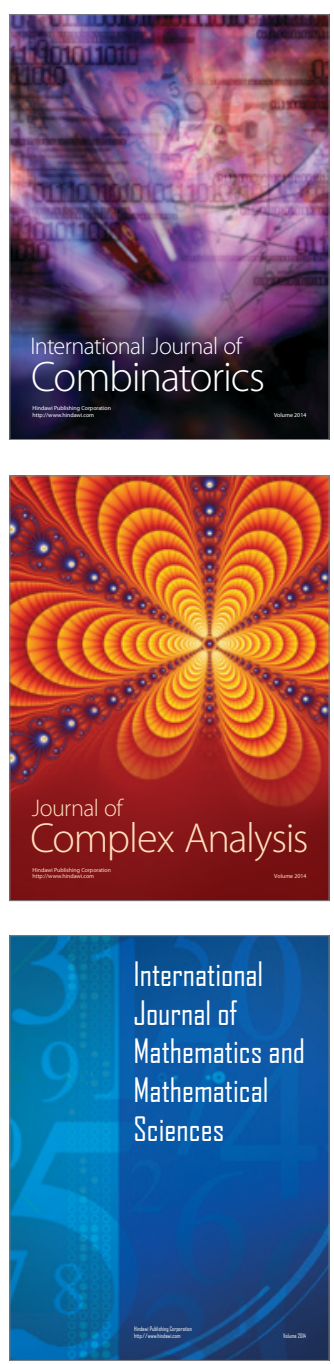
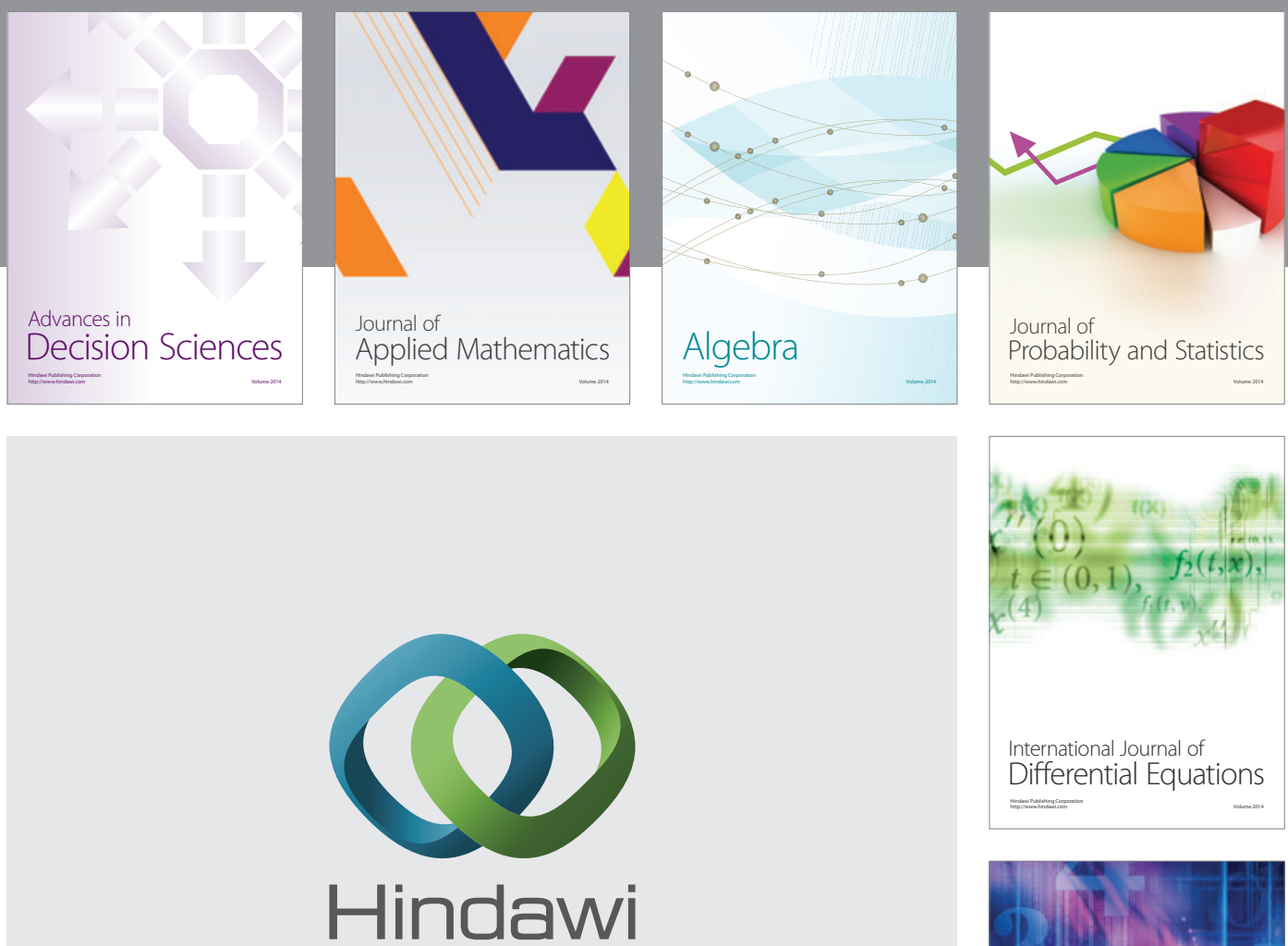

Submit your manuscripts at http://www.hindawi.com
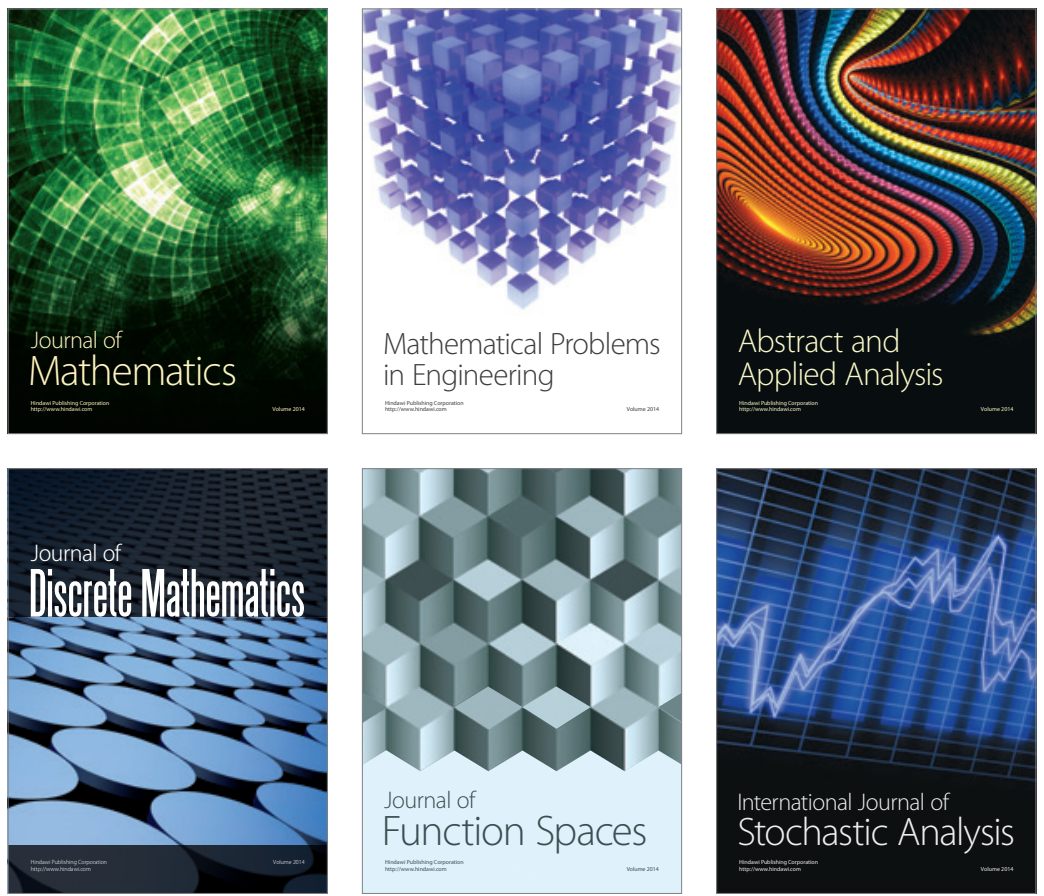

Journal of

Function Spaces

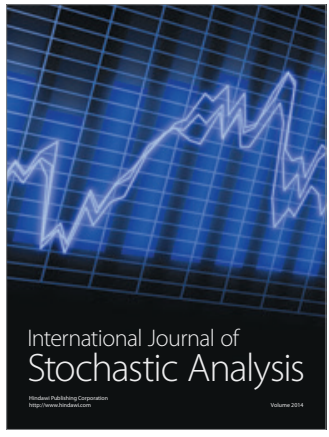

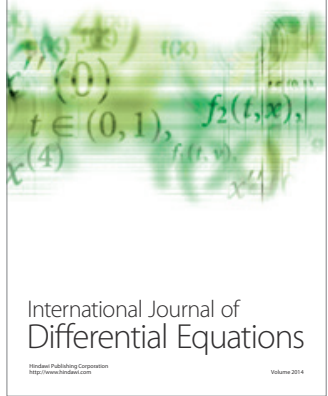
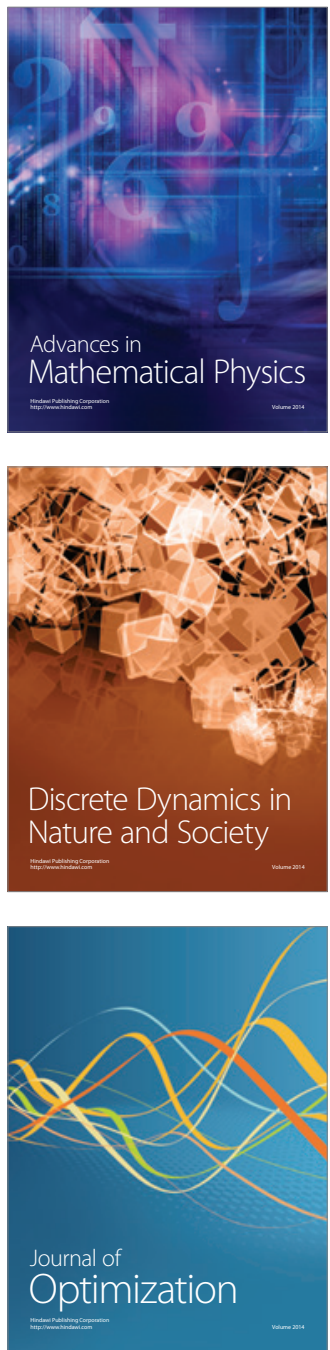